\title{
Culture and characterization of presumptive embryonic stem cell line isolated from New Zealand White rabbit embryos
}

Payungsuk Intawicha ${ }^{1}$, Yao-Wen $\mathrm{Ou}^{1}$, Su-Chun Zhang ${ }^{2}$, Hong-Lin $\mathrm{Su}^{3}$, Hwa-Fen Guu ${ }^{4}$, Ming-Jer Chen ${ }^{4}$, KunHsiung Lee ${ }^{5}$, Jyh-Cherng Ju ${ }^{1}$

${ }^{1}$ Department of Animal Science, National Chung Hsing University, Taichung; ${ }^{2}$ Waisman Center, University of Wisconsin, Madison, Wisconsin, USA; ${ }^{3}$ Department of Life Science, National Chung Hsing University, Taichung; ${ }^{4}$ Taichung Veterans General Hospital, Taichung; ${ }^{5}$ Animal Technology Institute Taiwan, Chunan, Miaoli

The objectives of this study were to establish and characterize rabbit ES cells lines. Blastocysts were collected from New Zealand White rabbits during Days 4 to 5 post-mating. After removal of the mucin coat and the zona pellucida by pronase, embryos were directly cultured in ES cell medium on the mitomycin C-treated mouse embryonic fibroblast (MEF) feeder. In trial 1, seventy-one blastocysts collected from five rabbits were randomly allocated to two treatment groups (with or without leukemia inhibitory factor, LIF). The primary ICM colonies were formed in $\mathbf{8 5 . 7 \%}$ (30/35) of the cultured ES cell in the medium supplemented with LIF and $91.7 \%$ (33/36) from that without LIF. Fiftyseven percent of those primary colonies $(20 / 35)$ were successfully grown into ES-like cell lines in medium with LIF group. However, no cell lines were established in the medium without LIF, because those presumptive ES cells lost their colonial morphology or started the differentiation. In contrast, long-term culture in medium containing LIF resulted in four rES cell lines maintain morphology of ES-like cells. One of them had more than twenty-five passages and another one past thirty-two passages. In trial 2, presumptive rES cell colonies were examined for different pluripotent markers. Fifteen ES cell lines expressed the alkaline phosphatase (AP) and ten of them expressed ES cell surface markers, SSEA-4 and Oct-4. Westernblot analysis also confirmed the expression of Oct-4 protein. By RT-PCR, the Sox2 gene expression was weakly detected in some cell lines. Three cell lines were further induced to differentiate into embryoid bodies and ectodermal development was detected by histological examination. Another four cell lines were injected to SCID mice and tumorigenesis with three germ layers was also detected. In conclusion, presumptive New Zealand White rabbit ES cells can be established using our current protocols which can be longterm cultured on MEF feeders in medium containing LIF. Those ES cells express pluripotent stem cell makers and maintain the ability to differentiate into different tissue cells. Differentiation of these cell lines into specific cell types, such as pancreatic cells for treatment of diabetes and other disease models is undergoing.

Keywords: embryoid body, ES cells, LIF, rabbits

Cell Research (2008) 18:s38. doi: 10.1038/cr.2008.128; published online 4 August 2008

Correspondence: Jyh-Cherng Ju

E-mail: jcju@dragon.nchu.edu.tw or jcju@1sc.net.tw 